\title{
Automatización de un proceso para la determinación de parámetros químico-analíticos de calidad en muestras de
}

\section{Automation of a process for the determination of chemical-analytical quality parameters in water samples}

\author{
Gabriela Cristina Chango Lescano. ${ }^{1}$, John Germán Vera Luzuriaga. ${ }^{2}$, Violeta Maricela \\ Dalgo Flores. ${ }^{3} \&$ Christiam Xavier Nuñez Zavala. ${ }^{4}$
}

Recibido: 10-04-2019 / Revisado: 15-05-2019 /Aceptado: 29-06-2019/ Publicado: 15-07-2019

\begin{abstract}
.
DOI: https://doi.org/10.33262/cienciadigital.v3i3.1.685

The knowledge of water quality is fundamental for its evaluation, processing and environmental management. For which there are widely detailed processes that allow analysis in laboratories, however, these methods established by legal regulations are usually long and tedious. For this reason, the objective of this research is to propose an alternative to the traditional methodology of routine analyzes in the laboratory. To this end, work has been done on the automation of a system that allows the detection of $\mathrm{pH}$ and redox potential simultaneously in water samples by combining microfluidic techniques of chemical processes and microscale automation. The proposed method has been coupled to potentiometric techniques and has been applied to water samples, the proposed method has also been compared with the use of 3D technology, obtaining a versatile system with an analysis frequency of 5 samples per hour, decreasing the analysis time and the waste obtained in half.
\end{abstract}

1 Escuela Superior Politécnica de Chimborazo, Facultad de Mecánica, Riobamba, Ecuador. gabriela.chango@espoch.edu.ec

2 Escuela Superior Politécnica de Chimborazo, Facultad de Mecánica, Riobamba, Ecuador. john.vera@espoch.edu.ec

3 Escuela Superior Politécnica de Chimborazo, Facultad de Ciencias, Riobamba, Ecuador. violeta.dalgo@espoch.edu.ec

4 Instituto Superior Tecnológico Carlos Cisneros, Riobamba, Ecuador. christiamne @ gmail.com 
Keywords: Automation, Chemistry, Water, Potentiometry, pH, Redox Potential.

\section{Resumen.}

El conocimiento de la calidad del agua es fundamental para su evaluación, procesamiento y gestión ambiental. Para lo cual existen procesos ampliamente detallados que permiten el análisis en laboratorios, sin embargo, estos métodos establecidos por las normativas legales suelen ser largos y tediosos. Por esta razón, el objetivo de esta investigación es proponer una alternativa a la metodología tradicional de los análisis de rutina en laboratorio. Para tal fin, se ha trabajado en la automatización de un sistema que permite la detección de $\mathrm{pH}$ y potencial redox simultáneamente en muestras de agua combinando técnicas microfluídicas de procesos químicos y automatización a microescala. El método propuesto se ha acoplado a técnicas potenciométricas y ha sido aplicado a muestras de agua, también se ha comparado el método propuesto con el uso de tecnología 3D obteniendo un sistema versátil con una frecuencia de análisis de 5 muestras por hora disminuyendo el tiempo de análisis y los residuos obtenidos a la mitad.

Palabras claves: Automatización, Química, Agua, Potenciometría, pH, Potencial Redox.

\section{Introducción.}

El deseo de mejorar la calidad de vida ha hecho que el control de la calidad y la conservación del medio ambiente sean temas a priorizar en la ciencia contemporánea. Por otro lado, para evaluar la incidencia de las distintas sustancias es necesario desarrollar metodologías analíticas apropiadas que permitan obtener un gran número de datos, con niveles de selectividad y sensibilidad cada vez más exigentes, en períodos de tiempo cada vez más cortos y con costos razonablemente bajos, por lo tanto, la simplificación, automatización y miniaturización son tendencias que están marcando la evolución de la Química. Este avance conlleva el crecimiento paralelo de la Química Analítica junto a los nuevos procesos tecnológicos, extendiendo su campo de aplicación desde la investigación química fundamental hasta los métodos analíticos más sofisticados empleados en la industria química, farmacéutica, biotecnológica, química medioambiental, entre otros.

En este sentido la miniaturización es una tendencia importante en la química analítica, que permite ventajas importantes en comparación con las estrategias convencionales, como la portabilidad, el bajo consumo de reactivos y la poca generación de residuos.

Como primeros pasos en el proceso de automatización a micro escala tenemos la contribución de Skeggs (L. Skeggs, 1957) quien introdujo el concepto de métodos automáticos de análisis ligándolo al concepto de sustitución de una operación o medida estática realizada manualmente por una operación y medida continua o secuencial. De esta manera implantó la 
primera técnica de análisis en flujo conocida como Flow Injection Analyses (SFA) que consta de una bomba peristáltica y un conjunto de tubos de plástico llamados manifold usados para conducir los líquidos a un detector. En 1975 J. Ruzicka y E. H. Hansen proponen la técnica de análisis por inyección de flujo (FIA) (R̉užička, 1975) en la cual los componentes básicos son prácticamente los mismos que en SFA, pero el régimen de flujo es laminar, reduciendo el peligro de mezcla de una muestra con otra intercalando un volumen determinado de la muestra en una corriente de líquido portador mediante una válvula de inyección. Esta técnica ha sido muy utilizada para análisis aguas, en 1990 Valcárcel y su grupo (Valcárcel, 1990) realizaron la determinación simultánea de $\mathrm{pH}$, alcalinidad y concentración iónica total en agua potable.

El pH se midió por medio de un electrodo combinado de $\mathrm{pH}$ insertado en el flujo portador. La alcalinidad y los iones totales fueron determinados por medio de valoración. En 1996 Weeks y Johnson (Weeks, 1996) proponen sistemas de inyección en flujo convencionales en los que se sustituye la bomba peristáltica por microbombas como unidades dispensadoras de líquido, sin embargo, no es hasta el año 2002 en que grupos de investigación de la Facultad de Farmacia de la Universidad de Oporto y del Centro de Energía Nuclear en Agriculturade Piracicaba (CENA) (Lapa, 2002) introdujeron este mecanismo como parte de un sistema automático funcionando como impulsor de líquidos y válvula de conmutación a la vez controladas desde un ordenador para entregar muestras de solución de un volumen constante.

Las características y potencialidades de los sistemas de bombeo múltiple los hacen muy atractivos para la implementación de procedimientos analíticos automatizados, pues en contraste con otros sistemas de inyección de flujo, donde el volumen muestreado es determinado por el tiempo de muestreo (para un caudal dado), el volumen de muestra en los sistemas multibomba se define por el número de pulsos y el volumen de impulsos. Los sistemas de flujo de bombeo múltiple se controlan de la misma manera que los basados en multicomutación, lo que permite la utilización combinada de bombas solenoides y válvulas solenoides mejorando así la versatilidad y capacidad analítica.

La sencillez, flexibilidad y facilidad de operación de los sistemas de flujo multibombeo permiten la implementación de pequeños sistemas analíticos compactos con bajo consumo de muestras y reactivos, lo que representa una valiosa alternativa para sistemas de flujo más convencionales que dependen de bombas peristálticas o de jeringa y unidades de inserción de muestras separadas.

Esta técnica se ha utilizado para el análisis de aguas (Fernandes, 2009) (Lavorante, 2005), de fórmulas farmacéuticas (Lima, 2003) y se ha demostrado su robustez y versatilidad para la inserción, mezcla y transporte de soluciones.

La mayoría de las aplicaciones se han limitado a determinaciones directas siendo la espectrofotometría (J. L. F. C. Lima, 2003) y la quimioluminiscencia (Marques, 2005) las 
técnicas de detección más utilizadas. En el 2014 V. Cerdà introduce la técnica de Chips miniaturizados para el análisis por inyección en flujo multijeringa (V. Cerdà, 2014). En estos últimos años los Chips han sido combinados con detección espectrofotométrica (Petrova, 2015) y espectrofluorimetría (Frizzarin, 2015) debido a características como la compacidad, la posición rígida permanente de los canales de procesamiento de muestras que aseguran la repetitividad de las manipulaciones microfluídicas y su compatibilidad con otros instrumentos.

Estas mismas características hacen que el bombeo múltiple sea muy atractivo de usar en mediciones de potenciometría, que a menudo requieren una mezcla de muestra-reactivo.

El método de análisis potenciométrico se basa en mediciones del potencial electromotriz del elemento utilizando electrodos selectivos de iones (ISE), que son capaces de realizar mediciones rápidas y selectivas de la concentración de analito. Dicho método de detección es no destructivo, lo que significa que la muestra se puede utilizar para un análisis adicional. La potenciometría ha sido ampliamente usada para determinar la calidad en muestras de agua (Mesquita, 2011) (Alpízar, 1996) (Mimendia, 2010).

Además, es el método recomendado por la OMS (OMS, 2003) para la determinación de pH y potencial redox de una muestra acuosa. $\mathrm{El} \mathrm{pH}$ es uno de los parámetros operativos de calidad del agua más importantes a determinar, su importancia es tal que por ejemplo, para una desinfección efectiva con cloruro, el pH debe ser menor que 8.

El pH óptimo variará de acuerdo con la composición del agua y la naturaleza de los materiales de construcción utilizados en el sistema de distribución, pero a menudo está en el rango de 6.5-9.5 (OMS, 2003).

Por otro lado, las mediciones del potencial de óxido-reducción reflejan el estado redox del agua en estado líquido incluidos los gases disueltos, como el oxígeno. Este potencial se puede determinar utilizando un electrodo de Potencial de Reducción de la Oxidación (ORP). Las mediciones redox no se realizan ampliamente mediante servicios de agua potable, sin embargo, la OMS afirma que una ORP de $650 \mathrm{mV}$ causa una desactivación de virus casi instantánea, lo que demuestra que es un indicador de la contaminación patógena del agua (OMS, 2003).

Las medidas potenciométricas se basan en el hecho de que existe una relación lineal entre el potencial eléctrico establecido entre el electrodo de referencia y el logaritmo de actividad de los iones en la solución. Esta relación se describe mediante la ecuación de Nernst:

$$
E=E^{0}+\frac{2.203 R T}{z F} \log (a)
$$

Dónde: $\mathrm{E}$ es el potencial en $\mathrm{mV}$ que existe entre el electrodo sensor y el electrodo de referencia, z es la carga de iones, log (a) es el logaritmo de la actividad del ion medido. El 
factor $2.303 \mathrm{RT} / \mathrm{F}$ tiene un valor teórico de $59 \mathrm{mV}$ a $25^{\circ} \mathrm{C}$. La actividad es equivalente a la concentración en solución diluida pero se vuelve cada vez más baja a medida que aumenta la fuerza iónica.

La integración de procesos analíticos en sistemas miniaturizados permite varias ventajas, tales como: la medición y mezcla de todas las soluciones, la sincronización exacta hasta la lectura de los datos y la protección de la mezcla contra la contaminación externa. El objetivo de este trabajo fue desarrollar un sistema automático y portátil para la determinación simultánea de $\mathrm{pH}$ y potencial redox en un proceso microfluídico con detección potenciomética.

\section{Metodologia.}

\section{Reactivos y estándares}

Se utilizó soluciones tampón estándar de pH 4, 7 y 9 (Scharlau) para la calibración del electrodo de $\mathrm{pH}$ y una solución tampón de ORP $700 \mathrm{mV}$ para el electrodo de potencial redox. Se tomaron muestras de agua de potable y de piscina de la Ciudad de Riobamba, transportándolas al laboratorio y se insertaron inmediatamente en el sistema miniaturizado sin ningún tratamiento previo para validar el sistema propuesto.

\section{Aparatos y software}

El sistema está constituido por un módulo MPFS (Sciware Systems SL, Bunyola, España) para impulsar y seleccionar soluciones a través del chip (Sciware Systems SL) constituido por microbombas y válvulas solenoides que vienen calibradas de fábrica de manera que por cada pulso dispensa un volumen prefijado de líquido. Como líneas de transmisión se usan tubos de PTFE (0,8 $\mathrm{mm}$ i.d.). Para las mediciones potenciométricas se usó un multi potenciómetro EMF 6 (Lawson Labs Inc., Pennsylvania, EE. UU). La adquisición de datos y el control del sistema analítico se realizaron a través del software AutoAnalysis (Sciware Systems S.L.). El pH se midió con un electrodo plano CRISON modelo 52-07, electrodo de vidrio combinado (Ag / $\mathrm{AgCl})$.

El potencial redox se midió con un electrodo combinado Ingold (Ref. 105053189) con anillo de platino y referencia $(\mathrm{Ag} / \mathrm{AgCl})$ saturado con $\mathrm{KCl}$, este electrodo tiene un cuerpo esférico. El MPFS y el potenciómetro múltiple se conectaron en serie a través de una RS232C a un computador para el control remoto del software.

El paquete de software AutoAnalysis (Sciware Systems SL, Bunyola, España) se utilizó para el control instrumental, así como para la adquisición y el tratamiento de datos. El protocolo de software básico es adaptable al ensamblaje instrumental individual mediante la incorporación de bibliotecas de enlaces dinámicos (DLL), que pueden comunicarse con los instrumentos ensamblados individualmente. En la Figura 1 se muestra el esquema del sistema automatizado. 
Figura 1. Sistema automátizado

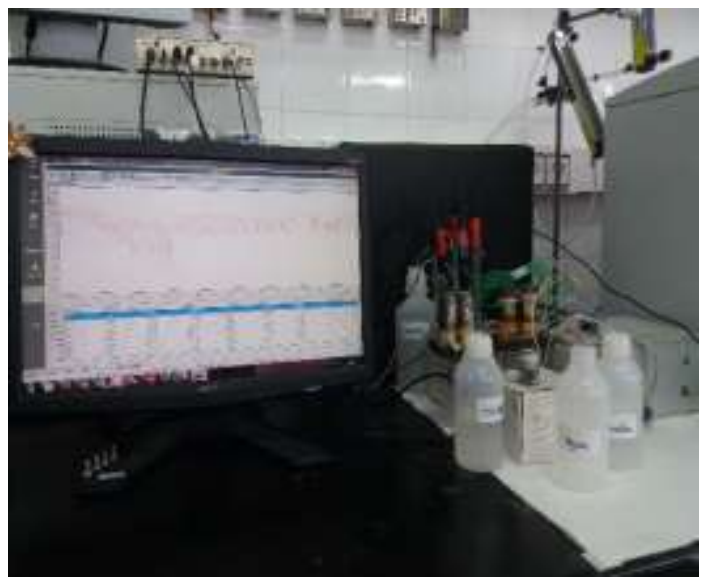

Fuente: Elaboración propia.

\section{Sistema y procedimiento de flujo}

El sistema automático se operó de acuerdo con 1 procedimiento descrito en la Tabla 1. El sistema comprende dos micro-bombas solenoide (B1 y B2) que fueron responsables de la inserción individual de la muestra y el agua destilada, además permitieron el control no solo de los volúmenes de solución, sino también del caudal, el tiempo y transporte desde la zona de toma de muestra hacia el detector. La secuencia de inserción de muestra y agua destilada estuvo a cargo de 2 micro válvulas solenoides que permitieron el paso de agua en posición OFF y de muestra en posición ON.

Tabla 1. Procedimiento automatizado para la determinación de $\mathrm{pH}$ y ptencial Redox a micro escala.

\begin{tabular}{rlll}
\hline \multicolumn{1}{c}{ Paso } & \multicolumn{1}{c}{ Descripción } & \multicolumn{1}{c}{ Acción } & Dispositivos activados \\
\hline 1 & Limpieza del sistema & Impulsar 1 $\mathrm{mL}$ de agua destilada & $\begin{array}{l}\text { B1, B2, V1 (OFF), V2 } \\
\text { (OFF) }\end{array}$ \\
2 & Adquisición de datos & -- & EMF 6 \\
3 & Activación de válvulas & -- & $\mathrm{V} 1(\mathrm{ON}), \mathrm{V} 2(\mathrm{ON})$ \\
4 & Determinación de pH & Impulsar 3mL of muestra & $\mathrm{B} 1$ \\
5 & Determinación de potencial redox & Impulsar 3mL of muestra & B2 \\
6 & Detener adquisición de datos & -- & EMF 6 \\
7 & Limpieza del sistema & Impulsar 1mL agua destilada & B1, B2, V1 (OFF), V2 \\
8 & Acondicionamiento del electrodo & Impulsar 3 $\mathrm{mL}$ solución tampon 7 y aire. & B1, B2 \\
\hline
\end{tabular}

Fuente: Elaboración propia.

En primer lugar, se impulsó $1 \mathrm{ml}$ de agua destilada a través del chip por la microbomba 1 y 2 (B1 y B2) para la limpieza del sistema con un caudal de $1 \mathrm{ml} \mathrm{min}{ }^{-1}$ (válvula 1 y 2 en 
posición OFF) (paso 1). El software AutoAnalysis activó el multi-potenciómetro EMF6 para la adquisición de datos (paso 2). A continuación, se activa las válvulas en posición ON para el análisis de la muestra (paso 3). Se insertó una alícuota de $3 \mathrm{~mL}$ en el chip por B1 y B2 desde la multibomba hacia los electrodos de $\mathrm{pH}$ y potencial redox simultáneamente (paso 4 y 5). El paso 1, 3 y 4 se repitieron tres veces, proporcionando tres mediciones repetidas. Después de eso, el software detuvo la adquisición de datos (paso 6). Se limpió el sistema con agua destilada (paso 7) para la siguiente medición. Finalmente, los electrodos se acondicionaron para almacenar el sistema (paso 8).

El tiempo total de análisis por muestra (con tres repeticiones) es de 12 minutos para la determinación simultánea de $\mathrm{pH}$ y potencial redox. Sería necesario entre 20 y 30 minutos para analizar ambos parámetros en una muestra manualmente. Esto incluye la limpieza de electrodos, calibración con soluciones tampón, cambio de muestras y análisis por triplicado de cada muestra. Es decir, el análisis de 5 muestras toma entre 2 y 3 horas para un técnico de laboratorio. La versatilidad del sistema diseñado consiste en utilizar el mismo chip para analizar diferentes parámetros simultáneamente. Además, se pueden analizar otros parámetros con el chip cambiando diferentes tipos de electrodos. Finalmente, el sistema está diseñado para analizar todos los tipos de muestras líquidas que no contengan sólidos en suspensión porque podrían bloquear los conductos dentro del sistema. En este caso, sería necesario enviar la muestra a un proceso de filtrado antes de ingresarla al sistema.

\section{Resultados.}

En el presente trabajo, la determinación de los parámetros $\mathrm{pH}$ y potencial redox fue definida por la mezcla dentro del reactor (Chip). Los resultados obtenidos con el chip de metacrilato usado en el sistema automático fueron comparados con un sistema sin mezclador incorporado y con un mezclador 3D impreso. Confirmando la importancia del mezclador dentro del sistema de flujo en cuanto a la mejor sensibilidad y linealidad obtenida (Figura 2).

Figura 2. Curvas de calibración de la comparación entre mezcladores

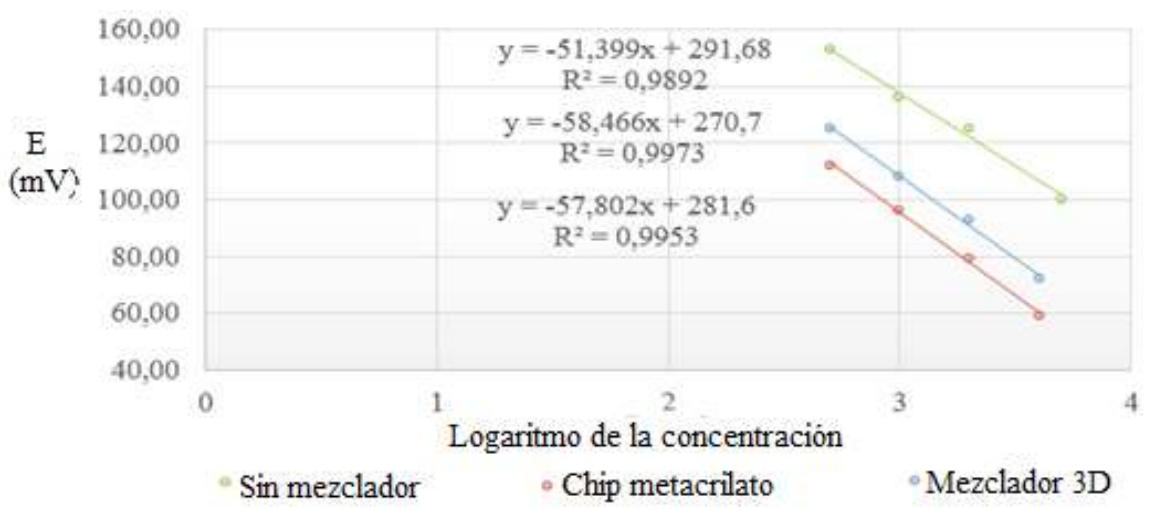

Fuente: Elaboración propia. 
El tiempo de estabilización del electrodo de vidrio para la determinación de $\mathrm{pH}$ en un proceso normal es alrededor de 1 minuto, sin embargo, a micro escala este tiempo incrementa notablemente entre 3 y 4 minutos para obtener curvas de calibración precisas. Los experimentos preliminares revelaron que la tasa de flujo óptima es de $2 \mathrm{ml} / \mathrm{min}$. Un valor alto produce un aumento indeseable en el límite inferior de la respuesta lineal, y para valores inferiores a $2 \mathrm{ml} / \mathrm{min}$ se reduce la tasa de muestreo. Soluciones tampón fueron usadas para la calibración del electrodo de $\mathrm{pH}$, los resultados obtenidos en los experimentos mostraron una pendiente de Nernstiana $(-53.5 \mathrm{mV} / \mathrm{pH})$ para un $\mathrm{pH}$ de 4 a 9 (Figura 3).

Figura 3. Curvas de calibración de medición de $\mathrm{pH}$

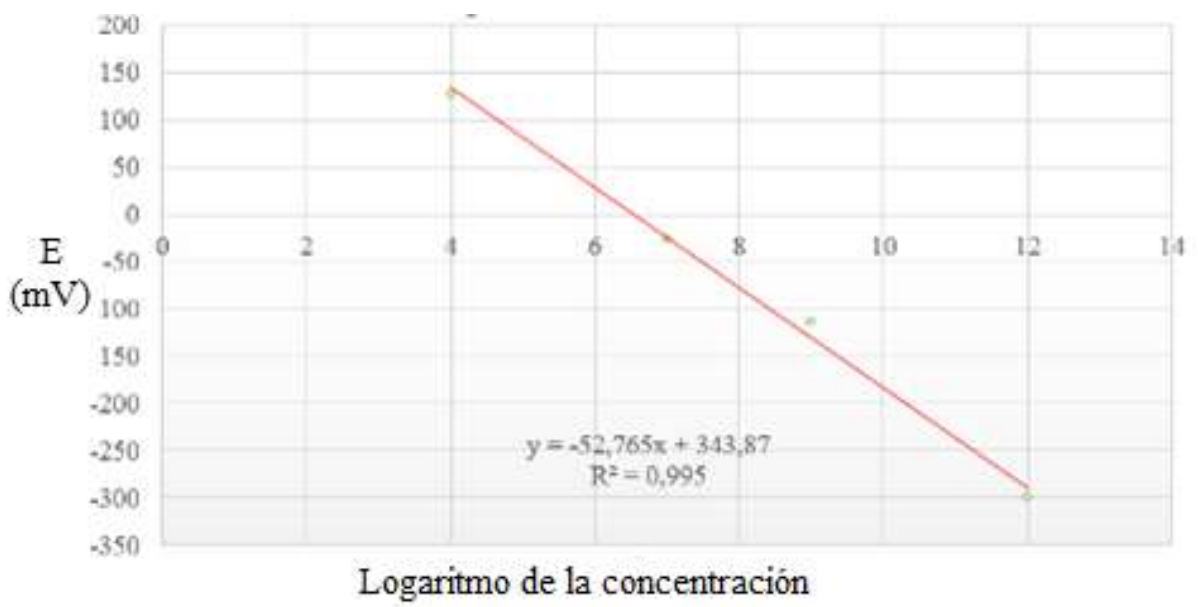

Fuente: Elaboración propia.

El electrodo de potencial redox fue calibrado con una solución tampón de ORP $700 \mathrm{mV}$, obteniendo una lectura estable a partir de los 3 minutos con un caudal de $2 \mathrm{ml} / \mathrm{min}$ (Figura 4).

Figura 2. Resultado de la lectura del electrodo de potencial redox.

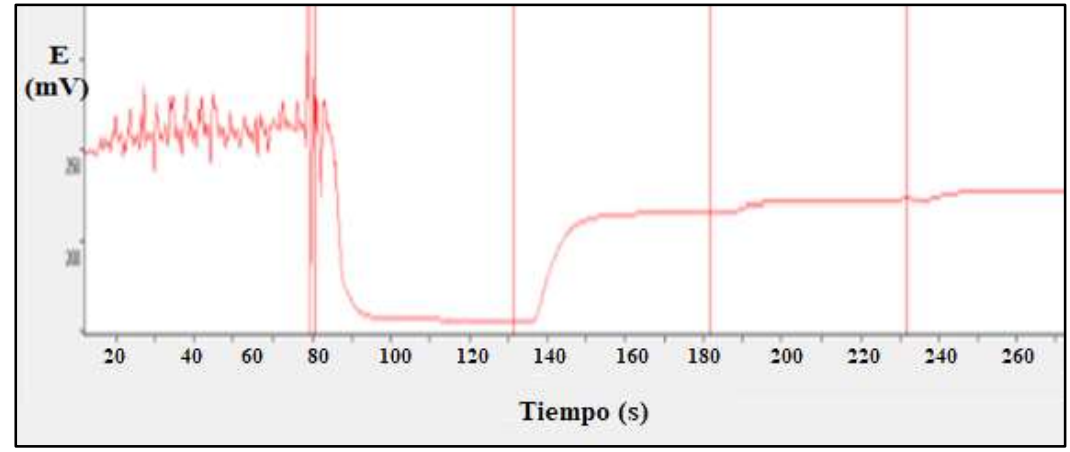

Fuente: Elaboración propia. 


\section{Validación y aplicación a muestras de agua.}

El sistema automatizado propuesto se ha validado mediante la comparación entre los resultados obtenidos con este sistema y el método convencional usado en laboratorio para la determinación del $\mathrm{pH}$ y potencial redox, dando resultados similares entre los métodos (Tabla 2).

Tabla 2. Resultados de la validación del método propuesto en muestras de agua

\begin{tabular}{lcccc}
\hline \multicolumn{1}{c}{ Muestra } & \multicolumn{2}{c}{$\mathbf{p H}$} & \multicolumn{2}{c}{ Potencial Redox } \\
\cline { 2 - 5 } & $\begin{array}{c}\text { Procedimiento } \\
\text { normal } \\
(\mathbf{m V})\end{array}$ & $\begin{array}{c}\text { Sistema } \\
\text { automático } \\
(\mathbf{m V})\end{array}$ & $\begin{array}{c}\text { Procedimiento } \\
\text { normal } \\
(\mathbf{m V})\end{array}$ & $\begin{array}{c}\text { Sistema } \\
\text { automático } \\
(\mathbf{m V})\end{array}$ \\
\hline Muestra 1 & $7,10 \pm 0,11$ & $7,40 \pm 0,14$ & $377 \pm 5$ & $202 \pm 8$ \\
Muestra 2 & $7,77 \pm 0,01$ & $8,02 \pm 0,06$ & $297 \pm 13$ & $207 \pm 9$ \\
Muestra 3 & $7,85 \pm 0,01$ & $8,01 \pm 0,38$ & $271 \pm 3$ & $213 \pm 7$ \\
Muestra 4 & $7,40 \pm 0,29$ & $8,12 \pm 0,30$ & $213 \pm 3$ & $213 \pm 7$ \\
Muestra 5 & $7,60 \pm 0,06$ & $7,91 \pm 0,10$ & $197 \pm 5$ & $211 \pm 5$ \\
Muestra de piscina & $7,30 \pm 0,05$ & $7,40 \pm 0,37$ & $610 \pm 8$ & $609 \pm 11$ \\
Buffer ORP & $1,53 \pm 0,04$ & $1,70 \pm 0,10$ & $700 \pm 16$ & $712 \pm 10$ \\
\hline
\end{tabular}

Fuente: Elaboración propia.

\section{Conclusiones.}

- En este trabajo se consiguió desarrollar un sistema automático para la determinación simultánea de $\mathrm{pH}$ y potencial redox mediante la combinación de técnicas microfluídicas, química analítica y sistemas de automatización, acopladas a un detector potenciométrico.

- Con el método propuesto y usando $2 \mathrm{ml}$ de muestra en un máximo de 9 minutos por muestra se logró determinar $\mathrm{pH}$ y potencial redox en el sistema previamente calibrado con tiempos de determinación menores que en el análisis de laboratorio tradicional.

- Los resultados fueron validados en muestra de aguas y piscina, por medio de la comparación de resultados con el método tradicional, sin encontrarse diferencias significativas. 


\section{Referencias bibliográficas.}

Alpízar, J. C. (1996). Simultaneous determination of chloride and fluoride ions in waters by sequential injection analysis. Electroanalysis, 1051-1054.

Fernandes, R. N.-R. (2009). Multi-pumping mechanised determination of selenium in natural waters by light emitting diode (LED) spectrometry. Journal of the Brazilian Chemical Society, 1242-1248.

Frizzarin, R. M. (2015). A portable multi-syringe flow system for spectrofluorimetric determination of iodide in seawater. Talanta, 1155-1162.

García-Cerda, L., Rodríguez-Fernández, O., Betancourt-Galindo, R., Saldívar-Guerrero, R., \& Torres-Torres, M. (2003). Síntesis y propiedades de ferrofluidos de magnetita. Superficies y Vacío., 16(1), 28-31.

J. L. F. C. Lima, S. M. (2003). Multi-pumping flow system for the spectrophotometric determination of dipyrone in pharmaceutical preparations. J. Pharm. Biomed. Anal, 1011-1017.

L. Skeggs. (1957). Automatic method for colorimetric analysis. Am. J. Pathol.

Lapa, R. A. (2002). Multi-pumping in flow analysis: concepts, instrumentation, potentialities. Analytica Chimica Acta, 125-132.

Lavorante, A. F.-R. (2005). Micro-pumping flow system for spectrophotometric determination of anionic surfactants in water. Analytical and bioanalytical chemistry, 1305-1309.

Lima, J. L. (2003). Multi-pumping flow system for the spectrophotometric determination of dipyrone in pharmaceutical preparations. Journal of pharmaceutical and biomedical analysis, 1011-1017.

Lin, Y.-S., Huang, K.-S., Yang, C.-H., Wang, C.-Y., Yang, Y.-S., Hsu, H.-C., Tsai, C.-W. (2012). Microfluidic synthesis of microfibers for magnetic-responsive controlled drug release and cell culture. PLOS ONE, 7(3), 1-8.

Marques, K. L. (2005). A catalytic multi-pumping flow system for the chemiluminometric determination of metformin. Analytical and bioanalytical chemistry, 452-457.

Mesquita, R. B. (2011). Development of flow injection potentiometric methods for the offline and on-line determination of fluoride to monitor the biodegradation of a monofluorop. Talanta, 1291-1297. 
Mimendia, A. L. (2010). Chemical Use of Sequential Injection Analysis to construct a potentiometric electronic tongue: Application to the multidetermination of heavy metals. Sensors and Actuators B: Chemical, 420-426.

OMS. (17 de 04 de 2003). Guías para la calidad del agua potable. Obtenido de https://www.who.int/water_sanitation_health/dwq/gdwq3_es_fulll_lowsres.pdf

Petrova, A. B. (2015). A miniaturized stepwise injection spectrophotometric analyzer. Analytical Sciences, 529-533.

Ṙužička, J. \&. (1975). Flow injection analysis. Analytica Chimica Acta, 145-157.

V. Cerdà, L. F. (2014). Flow Analysis A Practical Guide. Palma de Mallorca: Elsevier.

Valcárcel, M. \&. (1990). Flow injection analysis: A useful alternative for solving analytical problems. Fresenius' Journal of Analytical Chemistry, 662-666.

Weeks, D. A. (1996). Solenoid pumps for flow injection analysis. Analytical chemistry, 2717-2719. 


\section{PARA CITAR EL ARTÍCULO INDEXADO.}

Chango Lescano, G., Vera Luzuriaga, J., Dalgo Flores, V., \& Nuñez Zavala, C. (2019). Automatización de un proceso para la determinación de parámetros químico-analíticos de calidad en muestras de agua. Ciencia Digital, 3(3.1), 176-187. https://doi.org/10.33262/cienciadigital.v3i3.1.685

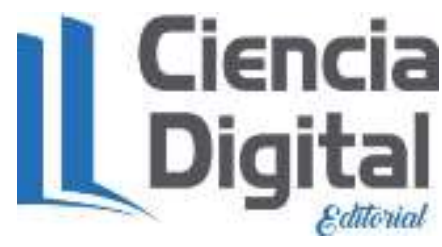

El artículo que se publica es de exclusiva responsabilidad de los autores y no necesariamente reflejan el pensamiento de la Revista Ciencia Digital.

El artículo queda en propiedad de la revista y, por tanto, su publicación parcial y/o total en otro medio tiene que ser autorizado por el director de la Revista Ciencia Digital.
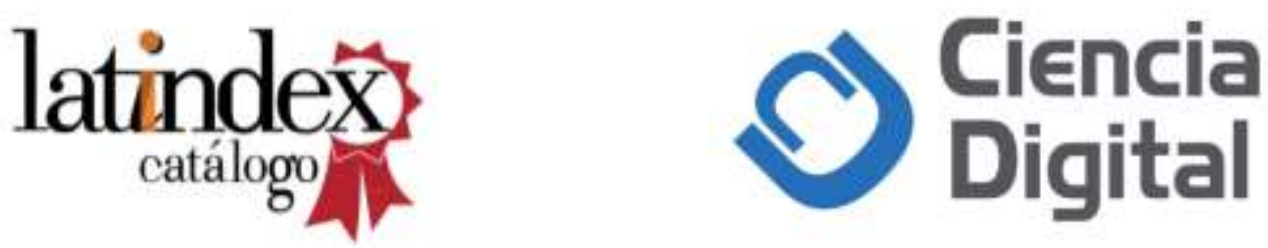